\title{
High obliquity, high angular momentum Earth as Moon's origin revisited by Advanced Kinematic Model of Earth-Moon System
}

Bijay K Sharma ( $\sim$ bksharma@nitp.ac.in )

Indian Institute of Technology

\section{Research Letter}

Keywords: Geosynchronous orbits, Earth's Obliquity Angle, Moon's Obliquity Angle, Lunar Plane Inclination, Lunar Orbit's Eccentricity, tidally interlocked

Posted Date: August 13th, 2020

DOI: https://doi.org/10.21203/rs.3.rs-54621/v1

License: (c) (i) This work is licensed under a Creative Commons Attribution 4.0 International License.

Read Full License 


\section{High obliquity, high angular momentum Earth as Moon's origin revisited by Advanced Kinematic Model of Earth-Moon System}

\section{Bijay Kumar Sharma ${ }^{1,2}$}

${ }^{1}$ Ex-Emeritus Fellow, National Institute of Technology, Patna 800005; email:

bksharma@nitp.ac.in, Phone:+919334202848.Affiliation for author 1.

2 Guest Researcher, Central Library, Indian Institute of Technology, Patna, Bihta

Campus,801106.Bihar,India.

Corresponding author: Bijay Sharma (bksharma@nitp.ac.in )

\section{Abstract}

Matija Cuk et.al (2016) have proposed a new model for the birth and tidal evolution of our natural satellite Moon, born from lunar accretion of impact generated terrestrial debris in the equatorial plane of high obliquity, high angular momentum Earth. This paper examines their findings critically in the light of Advanced Kinematic Model (AKM) which includes Earth's obliquity $(\phi)$, Moon's orbital plane inclination $(\alpha)$, Moon's obliquity $(\beta)$ and lunar's orbit eccentricity (e). It is shown that AKM's valid range of application is from $45 \mathrm{R}_{\mathrm{E}}$ to $60.33 \mathrm{R}_{\mathrm{E}}$. The evolution of $\alpha, \beta, \mathrm{e}$ is in correspondence with the simulation results of Matija Cuk et.al (2016) but evolution of Earth's obliquity has a break at $45 \mathrm{R}_{\mathrm{E}}$. According to $\mathrm{AKM}$, earlier than $45 \mathrm{R}_{\mathrm{E}}$ Earth should achieve $0^{\circ}$ obliquity in order to achieve the modern value of eco-friendly $23.44^{\circ}$ obliquity. Cuk et al (2016) silent on this point. AKM stands vindicated because using protocol exchange algorithm http://doi.org/10.1038/protex.2019.017, AKM has successfully given precise theoretical formalism of Observed LOD curve for the last 1.2Gy time span opening the way for early warning and forecasting methods for Earth-quake and sudden Volcanic eruptions. This paper gives us an algorithm to determine the short term and long term changes in Earth's 
obliquity which is related to Weather and Climate Extremes. Hence this paper gives us the mathematical tool for predicting the Earth's climate extreme.

\section{Keywords:}

Geosynchronous orbits; Earth's Obliquity Angle; Moon's Obliquity Angle; Lunar Plane Inclination; Lunar Orbit's Eccentricity; tidally interlocked;

\section{Introduction-Keplerian Era.}

The Kepler's Third Law for a given Planet-Sun configuration is:

$$
a^{3} \Omega^{2}=G(M+m)
$$

Eq. (1) does not specify if the given orbital configuration is stable. Newton derived this law assuming that centripetal force $\left(\mathrm{GMm} / \mathrm{a}^{2}\right)=$ centrifugal force $\left(\mathrm{mv}_{\text {tang }}{ }^{2} / \mathrm{a}\right)$ where $\mathrm{a}=$ semi-major axis of Earth-Moon orbital configuration, $\mathrm{M}=$ mass of the Earth and $\mathrm{m}=$ mass of our Moon. By implication it was assumed that all planetary configurations predicted by (1) are stable. By the end of $19^{\text {th }}$ century George Howard Darwin put a question mark on this stability by publishing two papers on E-M system (Darwin, 1879, 1880).

In $18^{\text {th }}$ Century, German Philosopher Kant had suggested the theory of retardation of Earth's spin based on the ancient records of Solar Eclipses (Stephenson \&Houldon, 1986; Stephenson 2003). Similar kind of studies had been carried out by Kevin Pang at Jet propulsion Laboratory at Pasadena (Morrison1978; Jong \& Soldt 1989). He happened to step upon certain ancient records regarding Solar Eclipses. A total Solar Eclipse had been observed in the town of Anyang, in Eastern China, on June 5, 1302 B.C. during the reign of Wu Ding. Had Earth maintained the present rate of spin, the Eclipse should have been observed in middle of Europe. This implies that in 1302 B.C. i.e. 3,291 years ago Earth's spin period was shorter by 0.047 seconds. This leads to a slowdown rate of 1.428 seconds per 100,000 years. 
In 1879 George Howard Darwin carried out a complete theoretical analysis of EarthMoon System and put forward a sound hypothesis for explaining the slowdown of Earth's spin on its axis. This marked the end of Keplerian Era. Gravitationally bound bodies were necessarily tidally interacting and tidal interaction led to tidal dissipation with inherent instability and hence a post-Keplerian physics was required to deal with gravitationally bound binary pairs. Tidally dissipative system because of loss of energy cannot be stable. The system will evolve to a minimum energy state which is a stable configuration by necessity.

\subsection{The beginning of Evolutionist view of Universe - Post-Keplerian Era.}

By mid $20^{\text {th }}$ century it was increasingly felt that celestial bodies pair behave as electrons orbiting the nucleus in individual atoms. Within an atom electrons had radiation-less stable permissible orbits propounded by Niels Bohr in 1913:

$$
\begin{gathered}
\text { Angular Momentum of electron }=I \times \omega=m \times r^{2} \times \frac{v_{\text {Tang }}}{r}=m \times v_{\text {Tang }} \times r \\
=n \times \frac{h}{2 \pi}
\end{gathered}
$$

Eq. (2) Simplifies to de Broglie standing wave condition:

$$
2 \pi r=n \times \frac{h}{m \times v_{\text {Tang }}}=n \times \frac{h}{p}=n \times \lambda_{\text {de-Broglie }}
$$

Eq.(3) Simply states that electrons are permitted to stay in radiation-less stable orbits where electrons behaves as matter wave which forms a Standing Wave and is inhibited from making synchrotron radiation and is ensured stable orbits. Any other orbit would collapse and electron would be launched on a death spiral towards its respective nucleus.

It is postulated by the Author that in exactly the same manner celestial body binaries are born at $\mathrm{a}_{\mathrm{G} 1}$ (inner Clarke's Orbit) which is a Keplerian Orbit , an equilibrium orbit where centripetal 
force is exactly balanced by centrifugal force but it is an energy maxima (Sharma 2011) hence the secondary tumbles short or tumbles long of $\mathrm{a}_{\mathrm{G} 1}$. In 2002 at World Space Cogress, Houston, Author proposed Kinematic Model(KM) (Sharma \& Ishwar 2002) . According to KM celestial body pairs have two triple synchrony orbits $\left(\mathrm{a}_{\mathrm{G} 1}\right.$ and $\left.\mathrm{a}_{\mathrm{G} 2}\right)$ where they are conservative systems and no dissipation of energy is involved (Sharma, Ishwar \& Rangesh,2009; Sharma, 2011; Krasinsky, 2002). Here triple synchrony orbits imply:

$$
\begin{gathered}
\omega(\text { spin angular velocity of the primary })=\Omega(\text { orbital angular velocity }) \\
=\Omega^{\prime}(\operatorname{spin} \text { angular velocity of the secondary }
\end{gathered}
$$

The orbits of triple synchrony are defined as geo-synchronous orbits in E-M system and Clarke's orbits in context of planet-satellite pairs, star-planet pairs, star-star pairs, neutron starneutron star(NS) pairs and NS and BH (black hole) pairs.

i. Planet-satellite pairs (with high mass ratio fractions above 0.2), star-planet pairs (with high mass ratio fraction above 0.2) and star pairs are non-relativistic systems. They, within months/years, lock-in at outer Clarke's orbit. Non-relativistic systems are stable at outer triple synchrony orbits.

ii. NS pairs, NS and BH pairs or BH pairs are relativistic systems. Relativistic systems are radiating gravitational waves and they are being driven towards coalescence hence they are always experiencing in-spiral orbital motion until the final ring-down and merger and always negatively off-setted with respect to outer Clarke's Orbit and never locked-in at outer Clarke's orbit. The magnitude of off-set is decided by the relativistic strength of NS pair/NS-BH pair/BH pair which in turn is decided by the rate of apsidal precession (long axis of the elliptical orbit of the secondary turning in the same direction as the secondary's orbital motion). 
iii. Tidally interacting binary pair such as planet-moon may have mass ratios from $10^{-4}$ to 0.2. These experience tidal evolution from inner Clarke's Orbit to Outer Clarke's orbit with time constant of evolution $\tau$ which is inverse power law of mass ratio $(q=$ secondary mass/ primary mass). As q decreases from 0.2 to $10^{-4}$ correspondingly time constant of evolution $\tau$ increases from year to Ky to My to Gy respectively (Sharma 2011).

iv. If mass ratio is infinitismal as in case Communication Satellite and Earth then time constant of evolution $\tau$ becomes infinite and Communication Satellite remains stay put in its orbit if it is shielded from Poynting Robertson Drag and radiation pressure.

\subsection{Two competing schools of thought on tidally interacting binaries.}

The whole scientific community had been using the elasto-viscous model for analyzing the tidally interacting binaries but this was based on the knowledge of Love number and Q factor of the celestial bodies which in turn depended on the knowledge of density, rigidity, viscosity and rate of periodic forcing. These parameters are known with large uncertainties for different Planets and their Satellites and hence their Tidal Evolutionary History will be arrived at with equal uncertainty in Seismic Model based analysis (Shi et.al. 2013). The Author developed a Kinematic Model (KM) of E-M system to study its tidal evolutionary history from its birth at inner geo-synchronous orbit $\left(\mathrm{a}_{\mathrm{G} 1}\right)$ to the final lock-in orbit at outer geo-synchronous orbit $\left(\mathrm{a}_{\mathrm{G} 2}\right)$ or to its final doom in glancing angle collision with Earth (Sharma 2011). The KM required only the globe-orbit parameters and the age of the system. Since the system parameters were known with high degree of confidence level hence the results arrived at were reliable and accurate. In spite of this improvement KM failed to resolve the conundrum in E-M puzzle. This had baffled the whole scientific community. 


\subsection{The conundrum of Earth-Moon puzzle.}

112 The Apollo mission had confirmed the age of E-M system with high degree of certainty as

113

114

115

116

117

118

119

120

121

122

123

124

125

126

4.467Gy (Stevenson 2008, Toubol et.al.2007). With this age of E-M system the present rate of recession of Moon should have been $2.4 \mathrm{~cm} / \mathrm{y}$ but Lunar Laser Experiment operational since $20^{\text {th }}$ July 1969 was giving a recession rate of 3.82 $\pm 0.07 \mathrm{~cm} / \mathrm{y}$ (Dickey et.al.1994) which indicated anomalously high dissipation rate in Earth's oceans and continents .If the present rate of recession was assumed and extrapolated into the past it led to the birth of Moon at 2.8Gy. In addition the $\mathrm{KM}$ with age as $4.467 \mathrm{~Gy}$ was not giving a matching theoretical formalism of observed LOD curve based on Coral fossils (Well 1963, 1966) and ancient tidalites (.Sonnett \& Chan 1997). The reconstruction of the history of Lunar recession from existing data of tidal rhythmites (Coughenour et.al. 2009) and that of length of Earth day (LOD) record in coral fossils ( Wells 1963,1966) indicate that Earth-Moon is not just a Clockwork or orbitally fixed pair of monoliths as viewed by Newton in Mathematica Principia but instead Earth-Moon system is a tidally evolving system where Moon is tidally receding from Earth since its birth. The findings of Stephenson (1997) and Lambeck (1980) firmly established that Earth-Moon system is a nonlinearly tidally evolving system with a complex history of interrupted tidal evolution due to intermittent gravitational resonances with lunar $\mathrm{M}_{2}$ tides and solar $\mathrm{S}_{2}$ tides . Kant (1754) hypothesis included the lunar tidal perturbation as well as solar tidal perturbation on tidal evolution of E-M system. G.H. Darwin $(1889,1890)$ clearly established that Moon's tidal brake and Solar tidal brake have slowed down the Earth's spin from 5 hrs and orbital radius of 18,000 Km (just beyond Roche's Limit) (Ida, Canup \& Stewart 1997) to the present Earth's spin rate of $24 \mathrm{~h}$ and orbital radius of 384,400 Km and in the process the Earth's angular excess spin energy released has led to tidal heating of Earth and spin angular momentum of Earth spin has been 
transferred to E-M orbital angular momentum and Lunar tidal recession. The results seem to indicate a non-linear variable rate of tidal dissipation throughout E-M system's history. Oceans may enter and exit $M_{2}$ and $S_{2}$ tide resonance in geologically short time intervals (Kagan 1997). It was this tension between observation and theory which compelled the Author to remove the first degree approximations from E-M model and propose a more comprehensive and detailed model with an elaborate dynamics of E-M system which the Author is referring to as Advanced Kinematic Mode;(AKM) and as will be shown in this paper AKM has dramatically improved the model to real world correlation.

\subsection{The origin of Moon from high obliquity, high angular momentum (AM) Earth and} impact generated circum-terrestrial debris disk.

$$
\text { Matija Cuk et.al (2016) have proposed a new model for the birth and tidal evolution of our }
$$
natural satellite Moon in which lunar tidal dissipation due to lunar obliquity tides during Cassini State transition plays an important role in stabilizing and allowing E-M system to arrive at climatically favorable E-M configuration with a low Earth's obliquity $\left(\phi=23.44^{\circ}\right)$. High angular momentum and high obliquity Earth provides a more robust mechanism to remove excess AM and provides Earth's mantle like isotopic composition properties of Moon.

Here there is a conundrum. As Moon's orbital plane inclination drops from $15^{\circ}$ to $5^{\circ}$, Earth's obliquity must rise from $0^{\circ}$ to $23.5^{\circ}$. This requires that at Cassini State Transition Earth's obliquity $\Phi$ must be 0 .

Rubicam (1993) has discussed this problem. At present, the Earth's mean obliquity is slowly increasing as a result of tidal interactions with the Moon. Simultaneously the lunar inclination is decreasing, so that the angular momentum of the Earth-Moon system is conserved.

The conservation of total angular momentum is given as follows (Rubicam 1993): 


$$
\operatorname{Sin}\left[\frac{\Delta \varphi}{2}\right]=-\frac{J_{\text {orbit }}}{J_{\text {Spin_Earth }}} \times \operatorname{Sin}\left[\frac{\Delta \alpha}{2}\right]
$$

157 This implies that if lunar orbital plane inclination angle decreases by $5^{\circ}$ and $\mathrm{J}_{\text {orbit }} / \mathrm{J}_{\text {spin_Earth }}=10$

158 then Earth's obliquity must increase by $60.6^{\circ}$. This precisely is predicted by Advanced

159 Kinematic Model as seen by close examination of Figure 1.

160

161

162

163

2 Materials and Methods

2.1. Calculation of Total Angular Momentum(AM) of Earth-Moon System as the vector sum of constituent AMs.

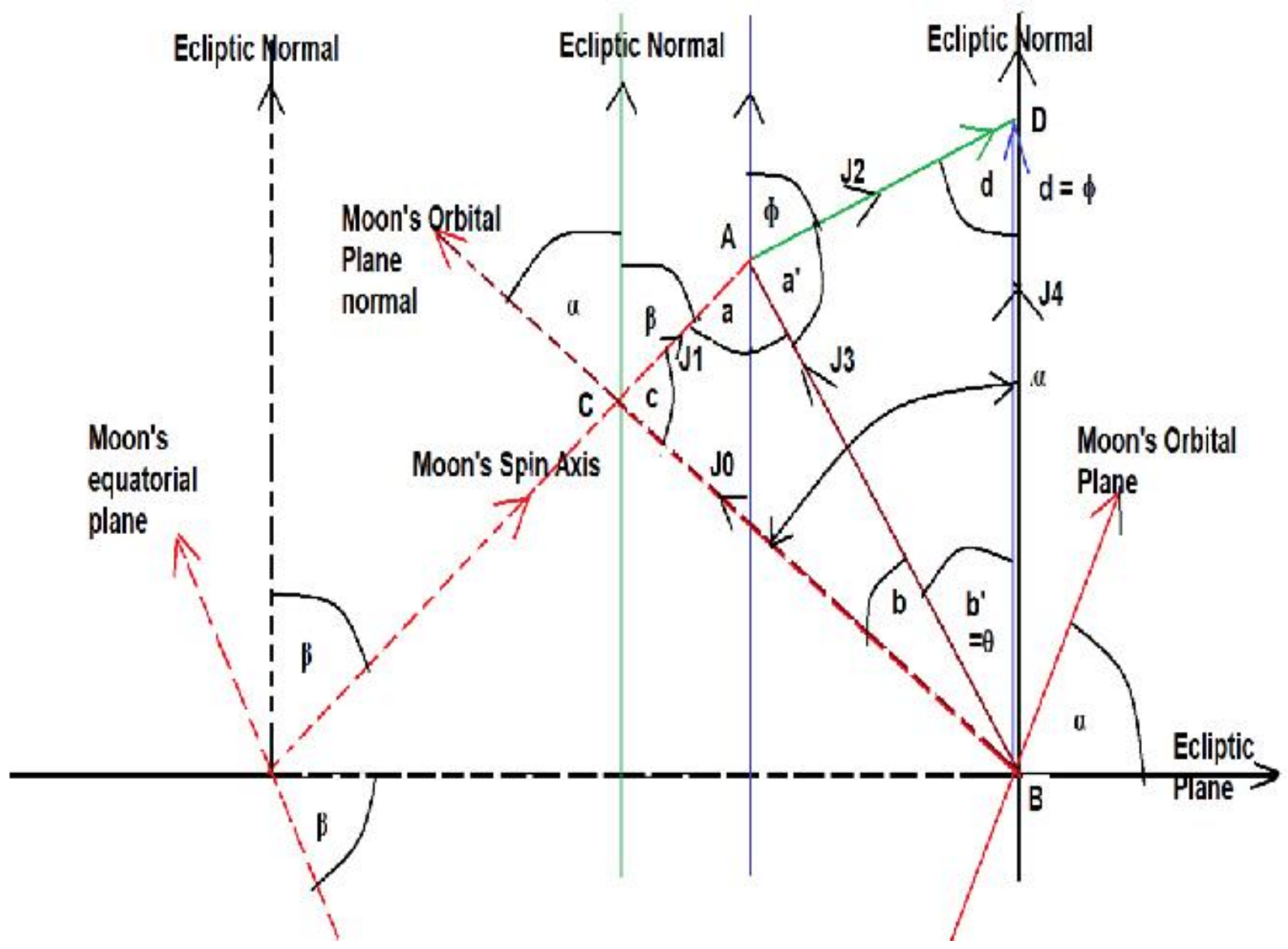

Figure 1. Spin-Orbital configuration of Earth-Moon System. 
In Figure $1, \mathbf{J}_{0}, \mathbf{J}_{1}, \mathbf{J}_{2}, \mathbf{J}_{3}, \mathbf{J}_{4}$, symbols are defined in List of Abbreviations.

$\mathbf{J}_{0}, \mathbf{J}_{1}$ and ecliptic normal are coplanar according to Cassini Law 3.

Before Cassini State transition E-M sysem was in Cassini State I when Moon's spin axis $\left(\mathrm{J}_{1}\right)$ and Moon's Orbital plane Normal $\left(\mathrm{J}_{0}\right)$ were on the same side of Ecliptic Normal. Presently E-M system is in Cassini State II hence $\mathrm{J}_{0}$ vector and $\mathrm{J}_{1}$ are on the two sides of Ecliptic Normal as shown in the Figure 1.

$\mathbf{J}_{3}, \mathbf{J}_{2}, \mathbf{J}_{4}$ and Ecliptic normal are coplanar.

But the plane containing $\mathrm{J}_{0}$ and $\mathrm{J}_{1}$ and Ecliptic Normal and the plane containing $\mathrm{J}_{2}, \mathrm{~J}_{3}, \mathrm{~J}_{4}$ and Ecliptic normal are two separate planes hence $\mathrm{J}_{0}$ and $\mathrm{J}_{1}$ are shown by dotted lines. Definitions of Earth's Obliquity $(\phi)$, Moon's orbital plane inclination $(\alpha)$ and Moon's Obliquity $(\beta)$ : Axial tilt of Earth's spin axis with respect to (w.r.t.) Ecliptic Normal $=\phi=23.44^{0}=$ 0.4091051767 radians;

Axial tilt of Moon's spin axis w.r.t. Ecliptic Normal $=\beta=1.54^{0}=0.02687807$ radians; Angle between Moon's equatorial plane and ecliptic plane $=\beta$; Total axial tilt of Moon's spin axis w.r.t. E-M orbital AM vector $=\alpha+\beta=6.68^{0}$ $=0.11658$ radians .

All these are observational Astronomy data in the current era and illustrated in Figure 1 and Figure S1.2 in S1 of supplementary materials.. The total resultant angular momentum vector $\left(\mathrm{J}_{4}\right)$ of Earth-Moon system is calculated in S1.1. of the supplementary materials.

First $J_{3}$ vector $=J_{0}$ vector $+J_{1}$ vector is calculated as shown in S1.2. 


$$
J_{3}=2.8498 \times 10^{34} \frac{\mathrm{Kg}-\mathrm{m}^{2}}{\mathrm{~S}}
$$

188 The angle of inclination of $\mathrm{J}_{3}$ w.r.t. ecliptic normal and left to normal $=\theta=\alpha-b=$

$189 \quad 0.08970905087$ radians $=5.13995^{\circ} \sim \alpha ;$

190 Next $\mathrm{J}_{4}$ vector $=\mathrm{J}_{3}$ vector $+\mathrm{J}_{2}$ vector $=$ Total $A M$ of $E-M$ system.

$$
\begin{aligned}
J_{\text {Total }}=J_{4}= & 3.37492 \times 10^{34} \frac{\mathrm{Kg}-\mathrm{m}^{2}}{\mathrm{~s}} \angle\left(\theta-b^{\prime}\right) \\
= & 3.37492 \times 10^{34} \frac{\mathrm{Kg}-\mathrm{m}^{2}}{\mathrm{~S}} \angle \gamma=0.39^{0}
\end{aligned}
$$

191 Next we determine the LOM/LOD equation, the cardinal equation of the Advanced Kinematic

192 Model.(The details of this derivation is given in S1 of the supplementary materials.

$$
\begin{gathered}
(N)^{2} \times a^{3}=X^{2}+\left(F \sqrt{1-k^{2}}\right)^{2} \times\left(a^{2}\right)^{2}+G^{2}+ \\
2\left(F \sqrt{1-k^{2}} \times a^{2}\right)(G)\left\{\sqrt{1-D^{2}} \sqrt{1-A^{2}}-A D\right\}+2 \times X \\
\times \sqrt{\left(F \sqrt{1-k^{2}} \times a^{2}\right)^{2}+(G)^{2}+2\left(F \sqrt{1-k^{2}} \times a^{2}\right)(G)\left\{\sqrt{1-D^{2}} \sqrt{1-A^{2}}-A D\right\}} \\
\times\left\{\sqrt{1-A^{2}} \sqrt{1-B^{2}}-A . B\right\} \\
X=\frac{\omega}{\Omega}=\frac{L O M}{L O D} ; B=\sqrt{G M+G m}=\sqrt{0.39860 \times 10^{6}+0.00490 \times 10^{6}} \\
=2.00873 \times 10^{7} \frac{m^{3 / 2}}{s} \\
N=\frac{J_{4}}{B \times C}=2.09517 \times 10^{-11}\left(\frac{1}{m^{\frac{3}{2}}}\right), \text { where } J_{4}=3.37492 \times 10^{34} \frac{\mathrm{Kg}-\mathrm{m}^{2}}{\mathrm{~s}} \\
C=8.01906 \times 10^{37} \mathrm{Kg}-\mathrm{m}^{2}, \\
G=\frac{I}{C}=0.00108949, \quad F=\frac{m^{*}}{C}=9 . .04936 \times 10^{-16}\left(\frac{1}{m^{2}}\right)
\end{gathered}
$$

193 Here we define the following Trignometric Identities: 


$$
\operatorname{Sin}[\alpha]=A ; \operatorname{Sin}[\beta]=D ; \operatorname{Sin}[\phi]=B
$$

194 These Trignometric Identities have been utilized in (8)

\section{3. Calculation of Earth Moon system parameters.}

196 3.1. Calculation of inner and outer geo-synchronous orbits in KM framework.

197 Our Moon has been born at inner geo-synchronous orbit, a $\mathrm{a}_{\mathrm{G} 1}$. E-M system has tidally evolved

198 from inner geo-synchronous orbit, $\mathrm{a}_{\mathrm{G} 1}$, to the outer geo-synchronous orbit, $\mathrm{a}_{\mathrm{G} 2}$. It is midway in

199 this evolutionary path at $\mathrm{a}=384,400 \mathrm{Km}$ from the center of the Earth. At both the geo-

200 synchronous orbit E-M system is in triple synchrony state when $\mathrm{X}=1$.

201 Equating $\mathrm{X}$ to Unity we obtain the two geo-synchronous orbits which has been done in S1

202 supplementary materials.

$$
\begin{aligned}
& a_{G 1}=1.48646 \times 10^{7} \mathrm{~m} \\
& a_{G 2}=5.33505 \times 10^{8} \mathrm{~m}
\end{aligned}
$$

203

204 Using Kepler's third law:

$$
\frac{1}{\Omega^{2}}=\frac{a^{3}}{B^{2}}
$$

205

210 sidereal day.

$$
\frac{L O M}{L O D}=\frac{\omega}{\Omega}=27.3217 \text { in modern times }
$$



it is being restated here:

$$
\begin{gathered}
(N)^{2} \times a^{3}=X^{2}+\left(F \sqrt{1-k^{2}}\right)^{2} \times\left(a^{2}\right)^{2}+G^{2}+ \\
2\left(F \sqrt{1-k^{2}} \times a^{2}\right)(G)\left\{\sqrt{1-D^{2}} \sqrt{1-A^{2}}-A D\right\}+2 \times X \\
\times \sqrt{\left(F \sqrt{1-k^{2}} \times a^{2}\right)^{2}+(G)^{2}+2\left(F \sqrt{1-k^{2}} \times a^{2}\right)(G)\left\{\sqrt{1-D^{2}} \sqrt{1-A^{2}}-A D\right\}} \\
\times\left\{\sqrt{1-A^{2}} \sqrt{1-B^{2}}-A \cdot B\right\}
\end{gathered}
$$

215 If real world is considered then:

216 Using current Earth's obliquity $\left(\Phi=23.44^{\circ}\right)$, current Moon's orbital inclination $\left(\alpha=5.14^{\circ}\right)$ and

217 current Moon's obliquity $\left(\beta=1.54^{\circ}\right)$ and $\mathrm{k}=0.0549$

218 We obtain the following trigonometric identities:

$219 \operatorname{Sin}[\alpha]=A=0.0895897$ and $\operatorname{Cos}[\alpha]=\sqrt{ }\left(1-A^{2}\right)=0.995979 ;$

$\operatorname{Sin}[\beta]=D=0.0268768$ and $\operatorname{Cos}[\beta]=\sqrt{ }\left(1-D^{2}\right)=0.999639$;

221

$$
\operatorname{Sin}[\Phi]=\mathrm{B}=0.397784 \text { and } \operatorname{Cos}[\Phi]=\sqrt{ }\left(1-\mathrm{B}^{2}\right)=0.917479 \text {; }
$$

$$
\left\{\sqrt{1-D^{2}} \sqrt{1-A^{2}}-A D\right\}=0.993211 \text { and }\left\{\sqrt{1-A^{2}} \sqrt{1-B^{2}}-A . B\right\}=0.87815
$$

222 Rewriting (8) and substituting the numerical values of the trigonometric identities in Modern

223 Times we get:

$$
\begin{gathered}
(N)^{2} \times a^{3}=X^{2}+(F \times 0.99849)^{2} \times\left(a^{2}\right)^{2}+G^{2}+ \\
2\left(F \times 0.99849 \times a^{2}\right)(G)\{0.993211\}+2 \times X
\end{gathered}
$$




$$
\begin{gathered}
\times \sqrt{\left(F \times 0.99849 \times a^{2}\right)^{2}+(G)^{2}+2\left(F \times 0.99849 \times a^{2}\right)(G)\{0.993211\}} \\
\times\{0.87815\} \\
.9
\end{gathered}
$$

224 Solving (9) with numerical values of N, F and G and ' $a$ ' (the current semi-major axis)

225 substituted we get the following quadratic equations:

$$
17826.5+234.495 X+X^{2}-24979.225=0
$$

226 The two roots of (10) are: -261.815 and 27.3199.

227 The negative root is rejected since both the spin of Earth and Moon and orbital motion are

228 retrograde. Hence only 27.3199 is tenable.

229 We are having a perfect match with observed LOM/LOD

230 (8) is satisfied for the current epoch $\omega / \Omega$ (LOM/lOD), $\alpha$ (Inclination angle), $\beta$ (lunar obliquity),

$231 \Phi$ (terrestrial obliquity) and e (eccentricity).

232 3.3. Evolution of inclination of Lunar orbital plane, eccentricity of Lunar orbit and

234 The empirical relation describing the evolution of Moon's orbital plane inclination with

235 respect to the ecliptic is (Supplementary-Information S2):.

$$
\begin{gathered}
\text { Inclination angle } \alpha=\frac{1.18751 \times 10^{25}}{a^{3}}-\frac{7.1812 \times 10^{16}}{a^{2}}+\frac{1.44103 \times 10^{8}}{a} \\
-8.250567342 \times 10^{-3}
\end{gathered}
$$

237 The empirical relation describing the evolution of Moon's obliquity angle ( $\beta$ ) is given as below 238 (Supplementary-Information S2):

Moon's Obliquity angle $\beta$

$$
=3.36402-1.37638 \times 10^{-8} a+1.32216 \times 10^{-17} a^{2}
$$


239 The empirical relation describing the evolution of Moon's orbit eccentricity is (Supplementary-

240 Information S2):

$$
e=0.210252+8.38285 \times 10^{-10} a-3.23212 \times 10^{-18} a^{2}
$$

\subsection{The Determination of the evolutionary history of Earth's Obliquity from Advanced}

\section{Kinematic Model of tidally interacting E-M system}

From a previous personal communication arXiv: $\underline{\text { htp://arXiv.org/abs/0805.0100 }}$

LOM/LOD of Earth Moon system is known over the tidal evolutionary history. It is tabulated in Table 1 of the Results.

Using (8)

$$
\begin{gathered}
(N)^{2} \times a^{3}=X^{2}+\left(F \sqrt{1-k^{2}}\right)^{2} \times\left(a^{2}\right)^{2}+G^{2}+ \\
2\left(F \sqrt{1-k^{2}} \times a^{2}\right)(G)\left\{\sqrt{1-D^{2}} \sqrt{1-A^{2}}-A D\right\}+2 \times X \\
\times \sqrt{\left(F \sqrt{1-k^{2}} \times a^{2}\right)^{2}+(G)^{2}+2\left(F \sqrt{1-k^{2}} \times a^{2}\right)(G)\left\{\sqrt{1-D^{2}} \sqrt{1-A^{2}}-A D\right\}} \\
\times\left\{\sqrt{1-A^{2}} \sqrt{1-B^{2}}-A . B\right\}
\end{gathered}
$$

Obliquity angle is determined.

In (8) all constant and all spatial functions are known except the obliquity angle $\Phi$.

For a given lunar orbit , LOM/LOD is known. Using this information $\operatorname{Sin}[\Phi]$ is determined and hence $\Phi$ and tabulated in Table 1.

We have six set of data from $\mathrm{a}=30 \mathrm{R}_{\mathrm{E}}$ to the present day semi-major axis.

In Supplementary-Information S1, the evolutionary history expression have been derived for LOM/LOD and Earth's obliquity $\Phi$ (radians). They are as follows: 


$$
\frac{L O M}{L O D}=\frac{\omega}{\Omega}=-12.0501+2.6677 \times 10^{-7} \times a-4.27538 \times 10^{-16} \times a^{2}
$$

$$
\phi=-0.732299+2.97166 \times 10^{-9} \times a
$$

256

260

261

262

263

264

\section{Results: The tidally evolving Earth-Moon System and its evolving astrometric} parameters.

Table 1 gives the evolutionary history of LOM/LOD and Earth's Obliquity.

Table 1. LOM/LOD and Earth's Obliquity for past geological epochs.

\begin{tabular}{|l|l|l|l|l|l|}
\hline $\mathrm{a}\left(\times \mathrm{R}_{\mathrm{E}}\right)$ & $\mathrm{a}\left(\times 10^{8} \mathrm{~m}\right)$ & $\mathrm{LOM} / \mathrm{LOD}$ & $\operatorname{Sin}[\Phi]$ & $\Phi$ (radians) & $\Phi^{\circ}$ \\
\hline 30 & 1.9113 & 23.3752 & -0.464076 & unstable & unstable \\
\hline 35 & 2.22985 & 26.1194 & -0.216896 & unstable & unstable \\
\hline 40 & 2.5484 & 28.1147 & 0.02137757 & 0.0213773 & 1.22483 \\
\hline 45 & 2.86695 & 29.2938 & 0.113547 & 0.113792 & 6.51 \\
\hline 50 & 3.1855 & 29.5965 & 0.218451 & 0.220227 & 12.6 \\
\hline 55 & 3.50405 & 28.9877 & 0.309749 & 0.314929 & 18 \\
\hline 60 & 3.8226 & 27.4 & 0.388198 & 0.398676 & 22.84 \\
\hline 60.335897 & 3.844 & 27.32 & 0.397788 & 0.409105 & 23.44 \\
\hline
\end{tabular}

We clearly see that at Cassini State Transition, Earth's obliquity is indeterminate. From $40 \mathrm{R}_{\mathrm{E}}$ to $60.336 \mathrm{R}_{\mathrm{E}}$ it is well behaved and obliquity is increasing. It increases from $1.22483^{\circ}$ to $23.44^{\circ}$.

This means that during angular momentum conservative phase reduction in inclination is accompanied with increase in obliquity by necessity. 
Table 2 gives the evolutionary history of $\omega / \Omega$ (LOM/1OD), $\alpha$ (Inclination angle), $\beta$ (lunar

266 obliquity), $\Phi$ (terrestrial obliquity) and e (eccentricity)

267 Table 2. evolutionary history of $\omega / \Omega(L O M / I O D), \alpha$ (Inclination angle), $\beta$ (lunar obliquity),

$\Phi$ (terrestrial obliquity) and e (eccentricity)

\begin{tabular}{|c|c|c|c|c|c|c|c|}
\hline $\mathrm{a}\left(\times \mathrm{R}_{\mathrm{E}}\right)$ & $\mathrm{a}\left(\times 10^{8} \mathrm{~m}\right)$ & $\omega / \Omega$ & $\alpha$ radians & $\beta$ & $\mathrm{e}$ & $\Phi(\mathrm{rad})$ & $\operatorname{Sin}[\Phi]$ \\
\hline 30 & 1.9113 & 23.3752 & $\begin{array}{l}0.480685 \\
\left(27.4^{\circ}\right)\end{array}$ & $\begin{array}{l}1.21635 \\
\left(69.69^{\circ}\right)\end{array}$ & 0.2524 & unstable & -0.464076 \\
\hline 35 & 2.22985 & 26.1194 & $\begin{array}{l}0.26478 \\
\left(15.17^{\circ}\right)\end{array}$ & $\begin{array}{l}0.952317 \\
\left(54.56^{\circ}\right)\end{array}$ & 0.236 & unstable & -0.216896 \\
\hline 40 & 2.5484 & 28.1147 & $\begin{array}{l}0.168969 \\
\left(9.68^{\circ}\right)\end{array}$ & $\begin{array}{l}0.71512 \\
\left(40.97^{\circ}\right)\end{array}$ & 0.214 & 0.0213773 & 0.0213757 \\
\hline 45 & 2.86695 & 29.2938 & $\begin{array}{l}0.124631 \\
( \\
\left.7.1408^{\circ}\right)\end{array}$ & $\begin{array}{l}0.504756 \\
\left(28.92^{\circ}\right)\end{array}$ & 0.1849 & $\begin{array}{l}0.113792 \\
\left(6.51^{\circ}\right)\end{array}$ & 0.113547 \\
\hline 50 & 3.1855 & 29.5965 & $\begin{array}{l}0.103801 \\
\left(5.04736^{\circ}\right)\end{array}$ & $\begin{array}{l}0.321225 \\
\left(18.4^{\circ}\right)\end{array}$ & 0.1493 & $\begin{array}{l}0.220227 \\
\left(12.6^{\circ}\right)\end{array}$ & 0.218451 \\
\hline 55 & 3.50405 & 28.9877 & $\begin{array}{l}0.0941394 \\
\left(5.39379^{\circ}\right)\end{array}$ & $\begin{array}{l}0.164527 \\
\left(9.4267^{\circ}\right)\end{array}$ & 0.10714 & $\begin{array}{l}0.314929 \\
\left(18^{\circ}\right)\end{array}$ & 0.309749 \\
\hline 60 & 3.8226 & 27.4 & $\begin{array}{l}0.0898729 \\
\left(5.149^{\circ}\right)\end{array}$ & $\begin{array}{l}0.03466 \\
\left(1.986^{\circ}\right)\end{array}$ & 0.0584 & $\begin{array}{l}0.398676 \\
\left(22.84^{\circ}\right)\end{array}$ & 0.388198 \\
\hline 60.336 & 3.844 & 27.32 & $\begin{array}{l}0.08971 \\
\left(5.14^{\circ}\right)\end{array}$ & $\begin{array}{l}0.0268 \\
\left(1.54^{\circ}\right)\end{array}$ & 0.0549 & $\begin{array}{l}0.409105 \\
\left(23.44^{\circ}\right)\end{array}$ & 0.397788 \\
\hline
\end{tabular}


In Figure 2, the evolution of Earth's obliquity $(\phi)$ based on AKM data (bold green) and based on

271 Simulation data (dashed green) by Mutja Cuk et.al(2016) is given. We see the discontinuity at $272 \quad 45 R_{\mathrm{E}}$.

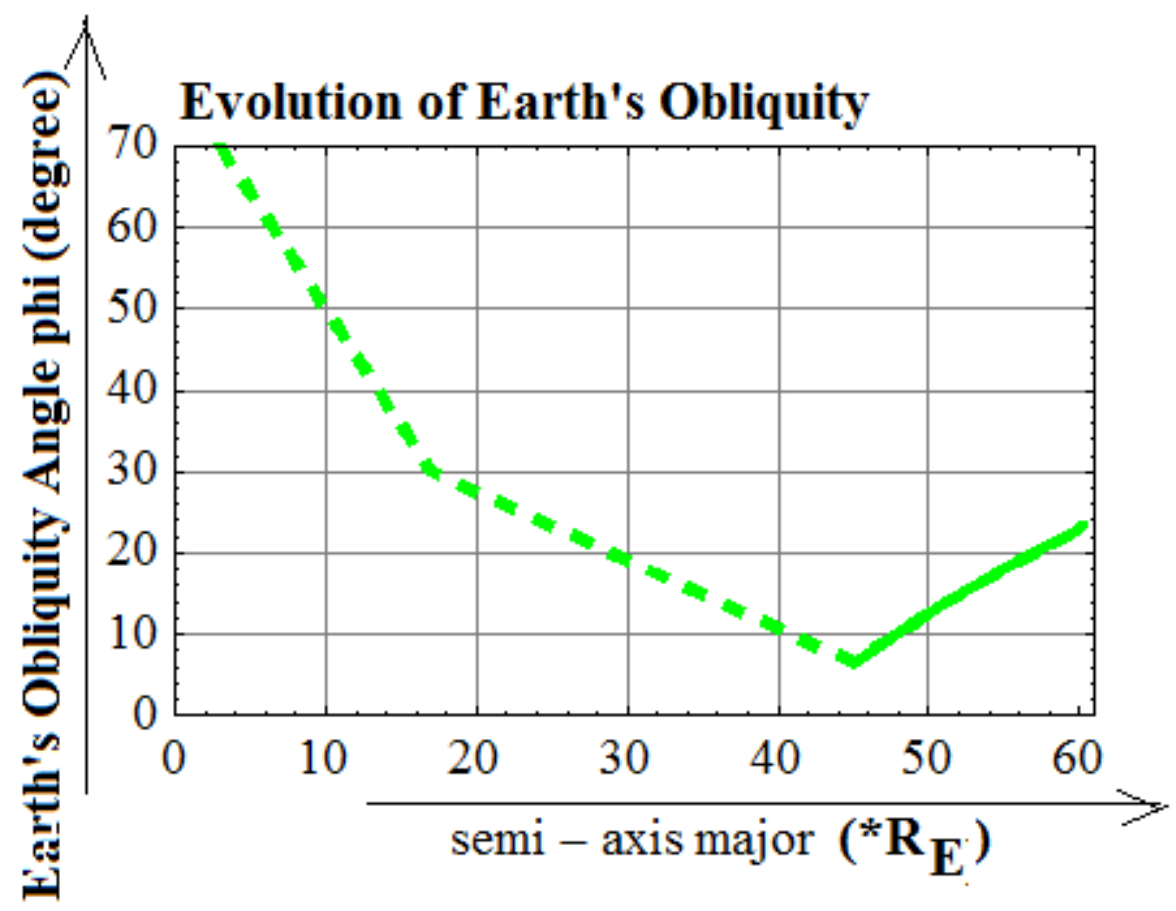

Figure 2. Earth's Obliquity angle $\left(\phi^{\circ}\right)$ evolution according to AKM (bold green) and according to Simulation results(dash green) (Cuk et.al.2016). 


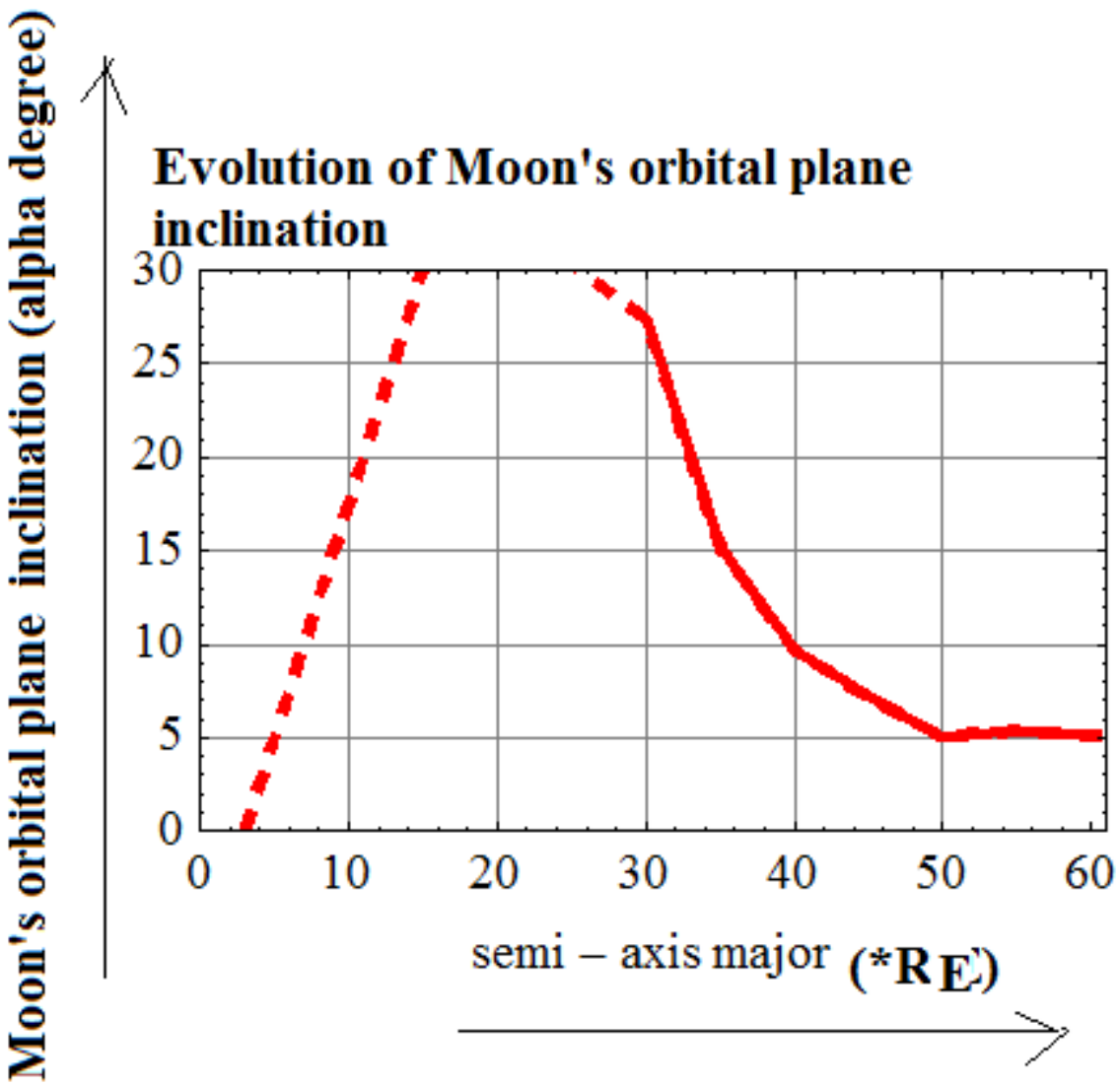
on Simulation (dashed blue) done by Matija Cuk et.al.(2016) is given. 


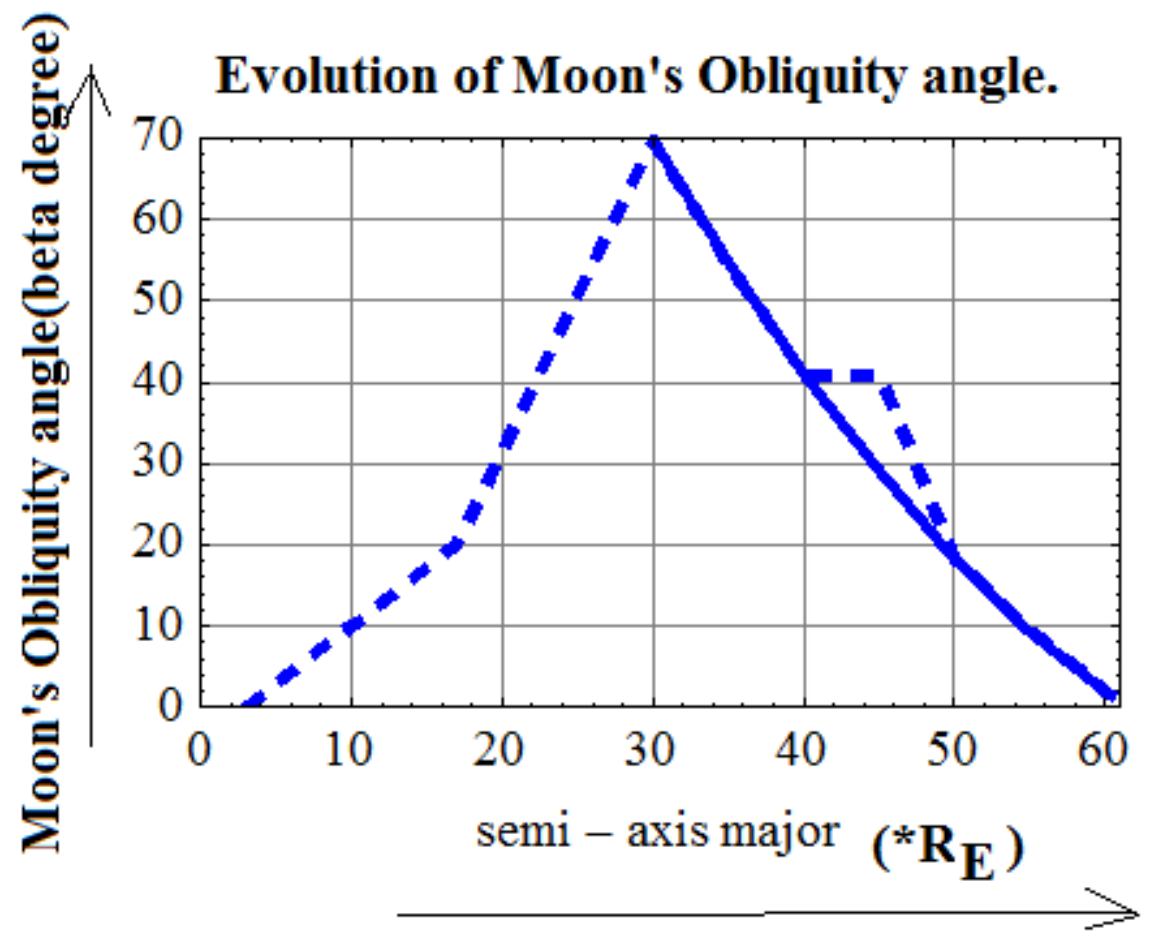

288 Simulation results (dash blue) (Cuk et.al.2016)

2895 Conclusions.

290 5.1. Discussion.

291 Examining the findings made by Cuk et.al and by this paper we see the following time line of 292 tidal evolution as tabulated in Table 3.

Table 3. Four stages in tidal evolution of E-M system.

\begin{tabular}{|l|l|l|l|l|l|}
\hline & Post- & Laplace Plane Transition & Cassini State & Cass. & Present \\
\hline ' $\mathrm{a}$ ' & $3 \mathrm{R}_{\mathrm{E}}$ & $17 \mathrm{R}_{\mathrm{E}}$ & Transition & State 2 & \\
\hline Ecc. & Circular & $0.5($ excess J drained to & 0.25 & $40 \mathrm{R}_{\mathrm{E}}$ & $60 \mathrm{R}_{\mathrm{E}}$ \\
\hline
\end{tabular}




\begin{tabular}{|l|l|l|l|l|l|}
\hline & orbit & heliocentric orbit) & & & \\
\hline$\alpha$ & 0 & $35^{\circ}$ & $28^{\circ}$ & $27.54^{\circ}$ & $5.14^{\circ}$ \\
\hline$\beta$ & 0 & $?$ & $69.69^{\circ}$ & $40.97^{\circ}$ & $1.54^{\circ}$ \\
\hline$\Phi$ & $70^{\circ}$ & $30^{\circ}$ & $?$ & $1.22^{\circ}$ & $23.44^{\circ}$ \\
\hline
\end{tabular}

295 Inspection of Table 3 leads us to a definite conundrum. After Cassini State 2 is reached, Earth-

296 Moon system enters angular momentum conservative phase. If lunar obliquity tides generated by

297 Cassini state transition help reduce inclination angle from $27.54^{\circ}$ to $5.14^{\circ}$. Then by necessity of 298 angular momentum conservation, obliquity must increase. This implies that current obliquity of $29923.44^{\circ}$ can be achieved only if obliquity angle is zero after Cassini State Transition.

300 This is obvious by the inspection of Figure 3, Figure 4 and Figure 5. As we see Figure 4 and 301 Figure 5 give a continuity between AKM results and Simulation results in the evolution of 302 inclination and Moon's obliquity data. The two results smoothly merge. But Figure 3 shows a 303 discontinuity near $40 \mathrm{R}_{\mathrm{E}}$ for Earth's obliquity. To achieve $23.44^{\circ}$ modern value of Earth's 304 obliquity the Earth Moon system must achieve $0^{\circ}$ Earth's obliquity just earlier than $40 \mathrm{R}_{\mathrm{E}}$ when 305 Moon settles down in Cassini state 2.

306 Since angular momentum conservation is not required from Laplace Plane Transition to Cassini 307 State Transition it is quite possible that strong obliquity tides are reducing inclination angle as 308 well as Obliquity angle. Then only the climate friendly low obliquity can be achieved.

309 At this point, Cuk et.al.(2016) are silent. This is a definite conundrum which needs to be 310 addressed before we can assert that 
311 "Our tidal evolutionary model supports high angular momentum, giant impact scenario to

312 explain Moon's isotopic composition and provide a new pathway to reach Earth's climatically

313 favourable low obliquity."

$314 \quad$ 5.2. Final summing up.

AKM will have to be critically examined in a sequel paper. Cuk Muteja et.al (2016) have proposed that Earth-Moon system while passing through Laplace plane transition and Cassini state transition pass through chaotic and turbulent phase and due to strong obliquity tides in Moon the tidal evolution gets stalled or even reversed for long periods of its existence. E-M system moves in 'Fits' from $3 \mathrm{R}_{\mathrm{E}}$ to $17 \mathrm{R}_{\mathrm{E}}$ and subsequently to $51.4 \mathrm{R}_{\mathrm{E}}$ in $3.267 \mathrm{~Gy}$ and then it 'Bounds' from $51.4 \mathrm{R}_{\mathrm{E}}$ to $60.33 \mathrm{R}_{\mathrm{E}}$ in $1.2 \mathrm{~Gy}$. At $17 \mathrm{R}_{\mathrm{E}}$ Laplace plane transition occurs and at $33 \mathrm{R}_{\mathrm{E}}$ Cassini state transition occurs. Cuk Matija have assumed that Moon is born from the Giant impact generated debris disk when Mars sized planetesimal made a glancing angle collision with proto Earth resulting in high obliquity and high Angular Momentum Earth. This resulted in isotopic identity of wide range of materials on Earth and Moon and the subsequent tidal evolution resulted in achieving climatically favorable Earth's obliquity of $23.44^{\circ}$. The application of AKM to this Fits and Bound model of E-M system gives a theoretical LOD curve which has precise match with observed LOD curve over last 1.2Gy as shown in SOM S6 using 
333 the Protocol Exchange algorithm http://org.1038/protex.2019.017 . In addition all the observed

334 performance parameters are theoretically justified. The observed parameters are LOD $=24 \mathrm{~h}$,

$335 \mathrm{LOM} / \mathrm{LOD}=27.32$ and velocity of recession of Moon as $3.82 \pm 0,07 \mathrm{~cm} / \mathrm{y}$ all these are a natural

336 corollary of AKM. Using the Protocol Exchange algorithim in Supplementary-On-line Materials

337 S6 AKM is vindicated on every count. In effect AKM has helped arrive at the correct theoretical

338 formalism of Observed LOD curve. This theoretical formalism will give the datum against which

339 the real time fluctuations in LOD will be compared and the precursors of the impending Earth-

340 quake and sudden volcanic eruptions will be identified and used to give Early Warning and

341 Forecasting for terrestrial disasters triggered by plate tectonic movements.

342 List of Abbreviations:

$343 \mathrm{~J}_{0}-$ orbital angular momentum of Earth-Moon system;

$344 \mathrm{~J}_{1}-$ spin angular momentum of Moon;

$345 \mathrm{~J}_{2}$ - Earth's spin angular momentum of Earth-Moon system;

$346 \quad \mathrm{~J}_{3}-$ vectorial sum of $\mathrm{J}_{0}$ and $\mathrm{J}_{1}$;

$347 \mathbf{J}_{4}-\mathbf{J}_{\text {Total }}-$ Resultant vectorial sum of angular momentums of Earth-Moon system;

$348 \quad \mathrm{KM}-$ Kinematic Model;

349 AKM - Advanced Kinematic Model;

350 E-M system - Earth-Moon System;

351 ' $\mathrm{a}$ ' - semi-major xis;

352 ' $\mathrm{a}_{\mathrm{Gl}}$ ' - inner geo-synchronous orbit;

353 ' $\mathrm{a}_{\mathrm{G} 2}$ ' - outer geo-synchronous orbit;

354 ' $\omega$ ' - spin angular velocity of Earth or Moon;

355 ' $\Omega$ ' - orbital angular velocity of Earth-Moon; 
$356 \quad$ NS - Neutron Star;

357 BH - Black Hole;

358 ' $\alpha$ ' - angle of inclination of Lunar Plane;

359 ' $\beta$ '-Moon's Obliquity;

360 ' $\phi$ ' - Earth's Obliquity;

361 Declarations:

362 Availability of Data:

363 Dataset for this Research has been generated in Section 3 and tabulated in Section 4 of the main

364 Text as well as it is tabulated in S2 file of the Supplementary Information.

365 The protocal for determining LOD in the past epoch is deposited at

366 http://doi.org/10.1038/protex.2019.017,

367 All the data and algorithm regarding LOM/LOD, LOD. Transit Time of Moon from higher

368 Energy Orbit to a lower Energy Orbit and astrometric parameters of Sun-Earth-Moon system

369 will be deposited in Open Topography Community Data Space once the access is obtained. Once

370 the access is obtained the link of the Public Repository will be provided.

371 Conflict of Interest:

372 I have no conflict of interest financial or otherwise whatsoever with anybody.

\section{Funding:}

374 Emeritus Fellowship Scheme sponsored by University Grants Commission, India. The Grant

375 number is EMERITUS/2012-13-GEN-855/.

376 Author's contribution:

$377 \quad$ i. $\quad$ In 1995 at $82^{\text {nd }}$ Session of India Science Congress I presented my first Research Paper $378 \quad$ titled 'Theoretical Formulation of Earth-Moon system revisited'. 
ii. In 1998 I got registered as D.Sc candidate in Post Graduate Mathematics Department, BRA Bihar University, Muzaffarpur, Bihar, India, under Prof. Bhola Ishwar.

iii. Since then this topic has been my valid primary research.

iv. I have published my research as Conference Proceedings

v. Proceedings in $34^{\text {th }}$ Scientific Assembly (2002) Houston, USA,

vi. Proceedings in $35^{\text {th }}$ Scientific Assembly (2004), Paris France,

vii. Proceedings in $39^{\text {th }}$ Scientific Assembly (2012) Mysore, India,

viii. in CELMEC V-2009 in Viterbo, Rome, Italy and

ix. in CELMEC VII-2017 in Viterbo, Rome, Italy

x. I also have two research articles in Advances in Space Research $(43,460-466,2009)$

xi. and in Earth, Moon and Planets (108,\#1,15-37,2011).

My three papers are under review:

A. in astronomy and computing: ASCOM-D-20-00005- precise theoretical formalism of observed LOD curve extending back to 1,2Gy.

B. ASCOM-D-20-00010- early demise of Phobos based on Architectural Design Rules.

C. 2020EF001728 - Past-Present and Future of Sun-Earth-Moon System in the Journal Earth's Future.

\section{Acknowledgments.}

I acknowledge the cooperation extended by the Director, IIT Patna for letting me use the resources at the central Library of the Institute. I acknowledge the cooperation extended by Prof. Pramod Kumar Mishra and Prof Pritam Kumar in EE Department, IIT Patna, for the use of their e-resources. in preparation of this paper. I also acknowledge the cooperation extended by the Director of NIT, Patna, as well as HOD, Electronics and Communication Department, NIT, Patna, for their continued support of my ongoing Research Programme in both Electronics and in Celestial mechanics.

\section{Author's Information:}

Author is affiliated with NIT Patna and IIT Patna with the objective of developing Early Warning and Forecasting Methods (EWFM) for Earthquakes and Sudden Volcanic Eruptions.

\section{References}

Atobe, K. and Ida, S.2007. “Obliquity evolution of extrasolar terrestrial planets”. Icarus 188,pp. 

1-17.

Barbanie, M.; Boehnke, P.; Kellar, B.; et.al.2017."Early Formation of the Moon 4.51 billon years ago”, Science Advances, 3, \# 1, e01602365, DOI: 10.1126/sciadv.1602365.

Burkhardt, C.2015. "Isotopic Composition of the Moon and the Lunar Isotopic Crisis", pp. 1-13 Published in Encyclopaedia of Lunar Sciences. Editor: Brian Cudnik, Affiliation:Prairie View A \& M University, Houston, USA. Copyright Information:Springer Nature, Switzerland, AG 2019, Publisher: Springer Cham, Online ISBN: 978-3-319-05546-6.

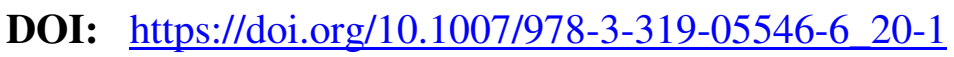

Coughenour, Christopher L.; Archer, Allen W.;Lacovaro, Kenneth J.;2009, “Tides, tidalites \& Secular Changes in the Earth-Moon System”, $\quad$ Earth-Science Reviews, 97, pp. 59-79, (2009).

Chyba, C. F., Jankowski, D. G. and Nicholson, P. D.1989. "Tidal evolution in the NeptuneTriton system”. Astron. Astrophys. 219, L23-L26 .

Cuk, M.; Hamilton, D. P.; Lock,S. J.; and Stewart, S. T.;2016. “Tidal evolution of of the Moon from high obliquity, high angular momentum Earth”, Nature, 539, pp. 402-406, http://doi.org/10/1038/nature19846 .

Canup, R. M. and Asphaug, E. 2001.“Origin of the Moon in a giant impact near the end of the Earth's formation”. Nature ,412,pp. 708-712 . https://doi.org/10.1038/35089010

David, T.J.; Hillenbrand, L. A,; Petigura, E. A.; Carpenter, J.M.; Crossfield, I.J.M.; Hinkley,S.:Ciardi,D.R.: Howard, A.W.:Issacson,H.T.;Cody,A.M.; Schlieder, J.E.; Beichman, C.A. and Barenfeld, S.A. 2016."A Neptune-sized transiting planet closely orbiting 5-10 million years old star”, Nature, 534, (7609),pp. 658-661, https://doi.org/10.1038/nature18293 
Dickey,J.O., Bender,P.L.,Faller,J.E., Newhall,X.X., Ricklefs,R.L.;Ries,J.G.; Shelus,P.J.; Veillet,C.;Whipple,A.L.;Wiant,J.R.;Williams,J.G.; and Yoder,C.F. 1994. 'Lunar Laser Ranging: A Continuing Legacy of the Apollo Program”, Science, 265, pp.482-490,; DOI: http://doi.org/10.1126/science.265.5171.482

Ida, S.: Canup, R. M.; Stewart, G.R.:1997, “Lunar Accretion from Impact generated disk”, Nature, 389, \# 6649, 353-357, 25 ${ }^{\text {th }}$ September 1997.

Kagan, B.A., 1997. "Earth-Moon tidal evolution: model results and observational Evidence". Progress in Oceanography 40, 109-124.

Kant, I., 1754. "Whether the Earth has undergone an alteration of its axial rotation", (Translated), Universal Natural History (1969). The University of Michigan Press, pp. 1-11.

Kaula, W. K. and Harris, A.1975 "Dynamics of Lunar Origin and Orbital Evolution," Review of Geophysics and Space Physics, 13, pp. 363..

Keane, J. T. and Matsuyama, I.,2014. "Evidence for Lunar true polar wander and a past low-eccentricity synchronous lunar orbit”, Geo. Phy. Letters, 41, 6610-6619, 2014, doi: http://doi.org/10.1002/2014GL061195

Lambeck, K., 1980. “The Earth's Variable Rotation; Geophysical Causes and Consequences”. Cambridge University Press. 449 pp

Nicholson, P. D., Cuk, M., Sheppard, S. S., Nesvorny, D. and Johnson, T. V.2008 "Irregular Satellites of the Giant Planets" pp.411-424.Publication: The Solar System Beyond Neptune, M.

Barucci, A.; Boehnhardt, H. ; Cruikshank, D. P.; and Morbidelli, A. (eds.), University of Arizona Press, Tucson, 592 Origin: LPI; Biblographic Code: 2008ssbn.book..411N 
456 Rubincam, D. P.1993 “The obliquity of Mars and “climate friction”. J. Geophys. Res. 98, $10827-10832(1993)$

Rubincam, D. P.2016..” Tidal friction in the Earth-Moon system and Laplace planes:

Darwin

$$
\text { redux”. Icarus 266, pp.24-43 . http://doi.org/10.1016/j.icarus.2015.10.024 }
$$

Sharma, B. K.1995 “Theoretical Formulation of Earth-Moon System revisited,” Proceedings of Indian Science Congress $82^{\text {nd }}$ Session, $3^{\text {rd }}$ January 1995 to $8^{\text {th }}$ January 1995 , Jadavpur University, Calcutta pp. 17.

Sharma, B. K. and Ishwar, B. 2002."Lengthening of Day curve could be experiencing chaotic fluctuations with implications for Earth-Quake Prediction”, World Space Congress-2002, $10^{\text {th }}-19^{\text {th }}$ October 2002, Houston, Texas, USA, Abstract 03078, Pg 3.

Sharma,B.K.and_Ishwar,B.2004A,“Planetary_Satellite_Dynamics:Earth-Moon,Mars Phobos-Deimos_and_Pluto-Charon_(Part-I)" $35^{\text {th }}$ _COSPAR_Scientific_Assembly,18$25^{\text {th }}$ July 2004A, Paris, France

Sharma, B. K. and Ishwar, B.2004B “A New Perspective on the Birth and Evolution of our Solar System based on Planetary Satellite Dynamics", $35^{\text {th }}$ COSPAR Scientific Assembly, 18$25^{\text {th }}$ July $2004 B$, Paris, France.

Sharma, B. K. and Ishwar, B. 2004C, “Jupiter-like Exo-Solar Planets confirm the Migratory Theory of Planets" Recent Trends in Celestial Mechanics-2004, pp.225-231, BRA Bihar University, $1^{\text {st }}-3^{\text {rd }}$ Novermber $2004 C$, Muzaffarpur, Bihar.Publisher Elsevier. 
Sharma,B.K., Ishwar, B. and Rangesh, N.2009 " Simulation software for the spiral trajectory of our Moon”, Advances in Space Research,43 (2009),460-466. https://doi.org/10.1016/j.asr.2008.07.001

Sharma, B. K. ;2011. “The Architectural Design Rules of Solar System based on the New Perspective", Earth, Moon and Planets, 108 (2011), pp. 15-37. DOI: https://doi.org/10.1007/s11038-010-9369-9

Shi, X.;Willner, K.; and Oberst, J.(2013).“Evolution of Phobos’ Orbit, Tidal Forces, Dynamical Topography, and Related Surface Modification Processes”. 44th Lunar and Planetary Science Conference (2013) 1889.pdf.

Sonett, C. P. and Chan, M. A.1998. "Neoproterozoic Earth-Moon Dynamics : rework of 900 million ago Big Cottonwood Canyon tidal laminae" Geophysics Research Letters, 25(4), 539-542. (1998).

Stephenson, F.R., 1997. “Historical Eclipses and Earth's Rotation”. Cambridge University Press. 576 pp.

Stevenson, J.David, 2008, “A planetary perspective on the deep Earth”, Nature,451, 261-265, (17 $7^{\text {th }}$ January 2008).

Toubol,M.; Kliene, T.; Bourdon, B.; Palme, H.;\&Wieler,R.;2007, “Late Formation and prolonged differentiation of Moon inferred from W- isotopes in lunar metals", Nature, 450, 1206-1209 (20 ${ }^{\text {th }}$ December 2007).

Wang, H.; Weiss, B. P.; Bai, X.; Downey, B.G. Wang,Jun; Wang, Jiajun; Suavet,C.; Fu,R.R.; Zucollotto, M.E. 2017. "Lifetime of the solar nebula constrained by meteorite paleomagnetism”, Science, 355, pp.623-627, DOI: http://doi,org/10.1126/science.aaf5043 
499 Ward, W. R.1975. "Past orientation of the lunar spin axis”. Science 189, pp.377-379.

500 Wells, John W.1963., “Coral Growth and Geochronometry”, Nature, 197, pp.948-950.

501 Wells, John W.,1966 “Paleontological Evidence of the Rate of the Earth's Rotation”, Earth-

502 Moon System, edited by Marsden \& Cameron, Plenum Press, pp. 70

503 Young, E. D.: Kahl,I.E.;Warren,P.H.;Rubia,D.C.;Jacobson,S.A.;and Morbidelli,A. .2016

504 "Oxygen isotopic evidence for vigorous mixing during the Moon-forming giant impact",

505 Science 351, 493-496. DOI: http://doi.org/10.1126/science.aad0525 
Figures

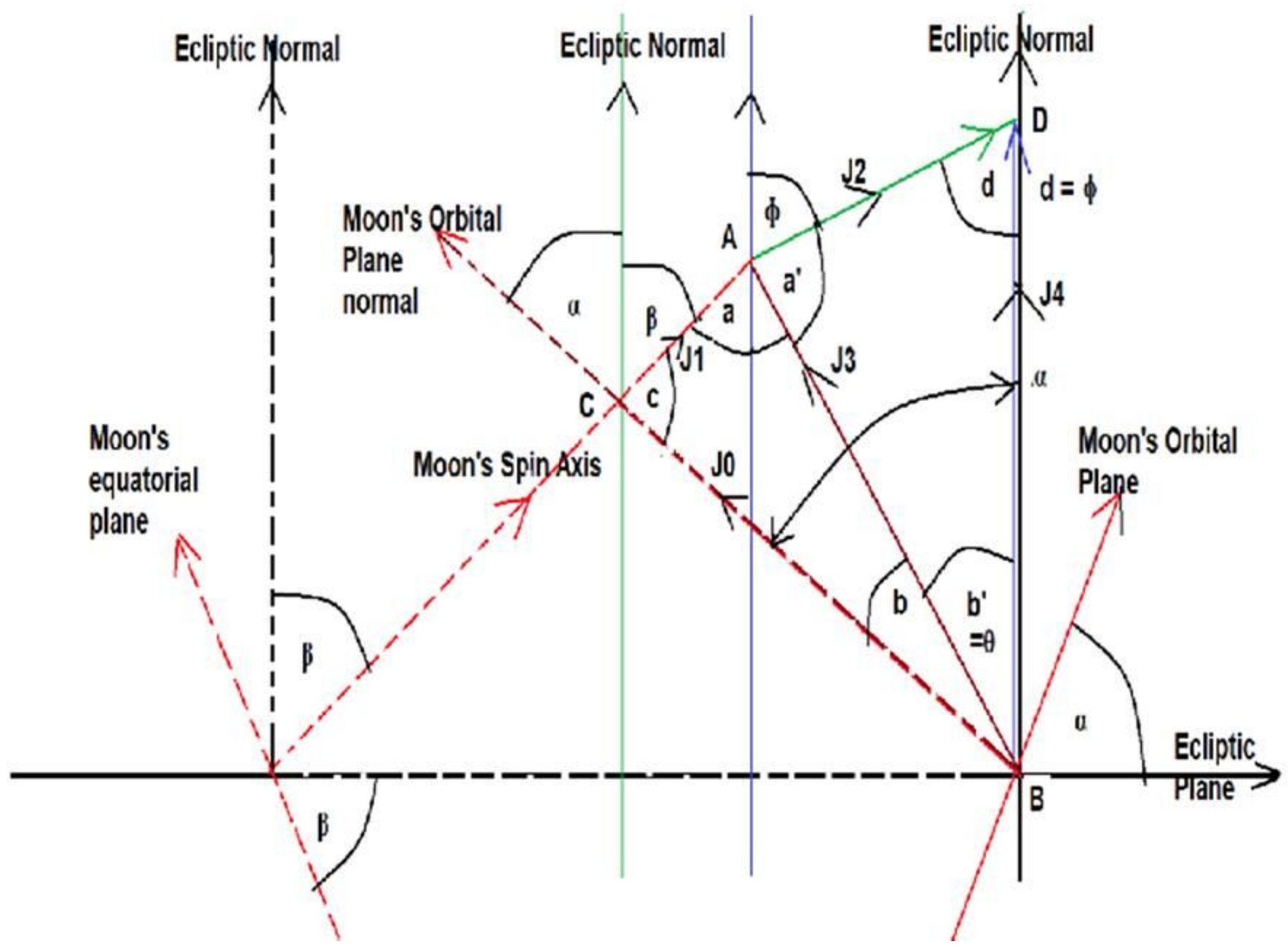

Figure 1

Spin-Orbital configuration of Earth-Moon System. 


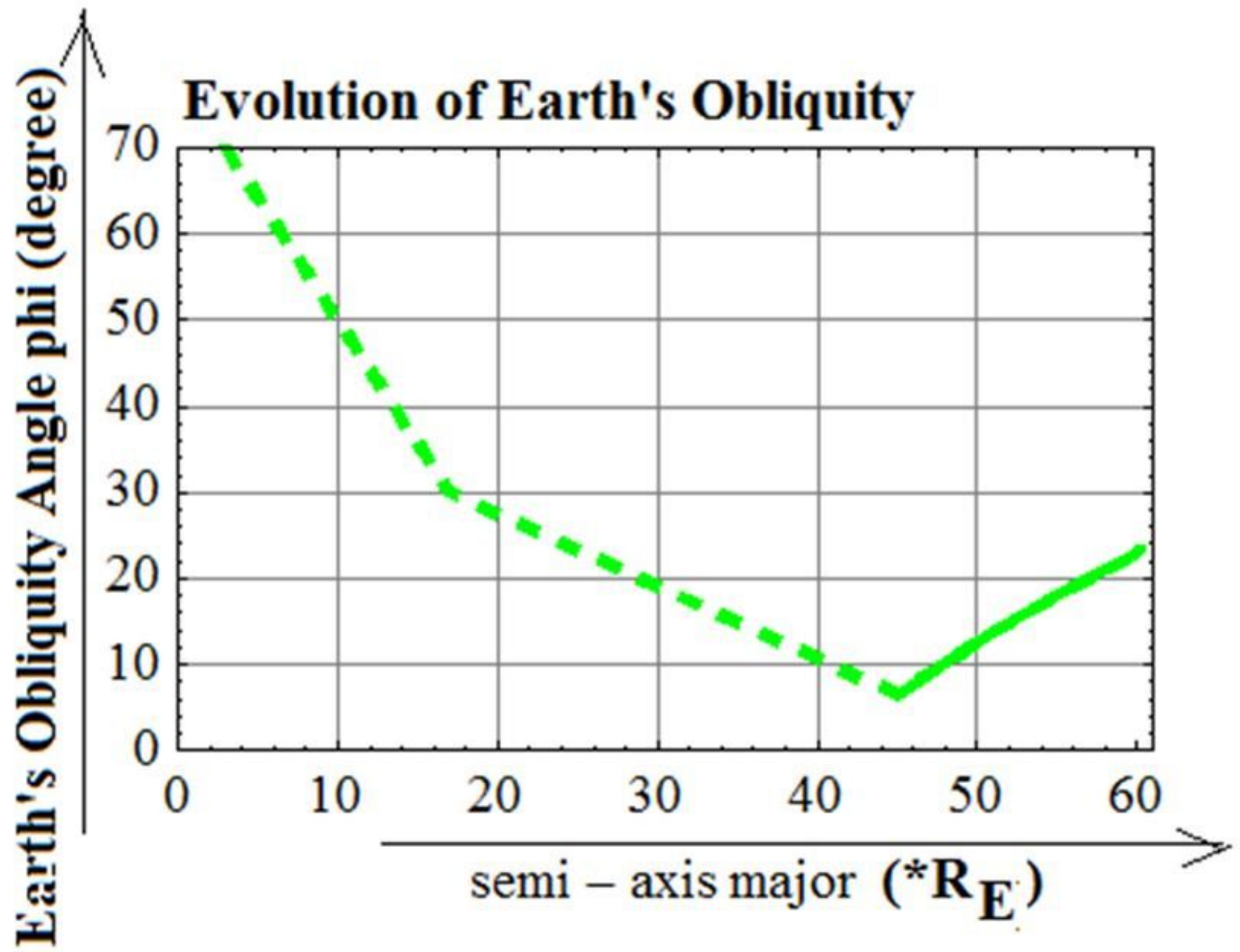

Figure 2

Earth's Obliquity angle $\left(\nabla^{\circ}\right)$ evolution according to AKM (bold green) and according to Simulation results(dash green) (Cuk et.al.2016). 


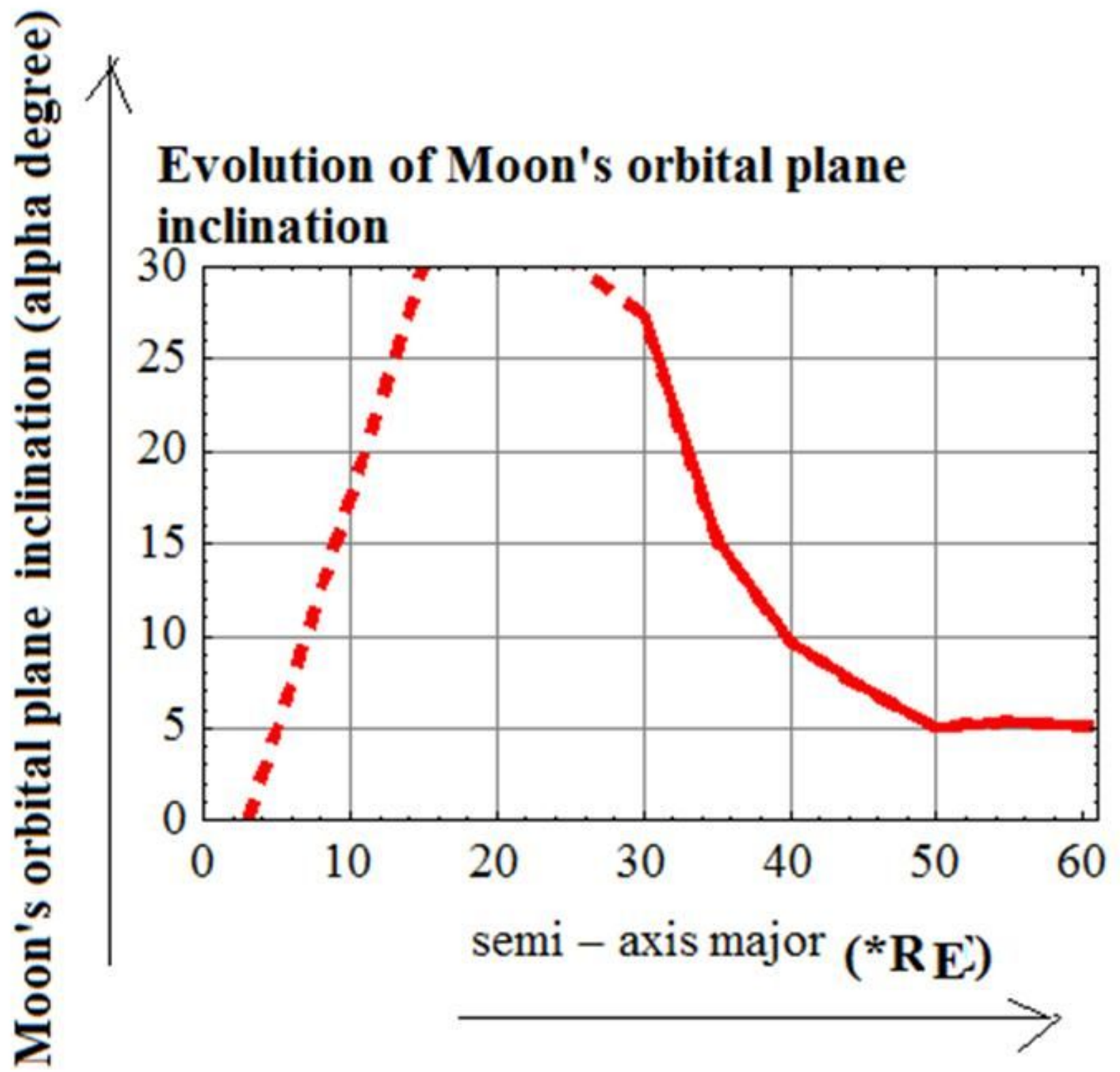

Figure 3

Moon's orbital plane inclination $\left(a^{\circ}\right)$ based on AKM (bold red) and based on Simulation results (dash red) (Cuk et.al.2016) 


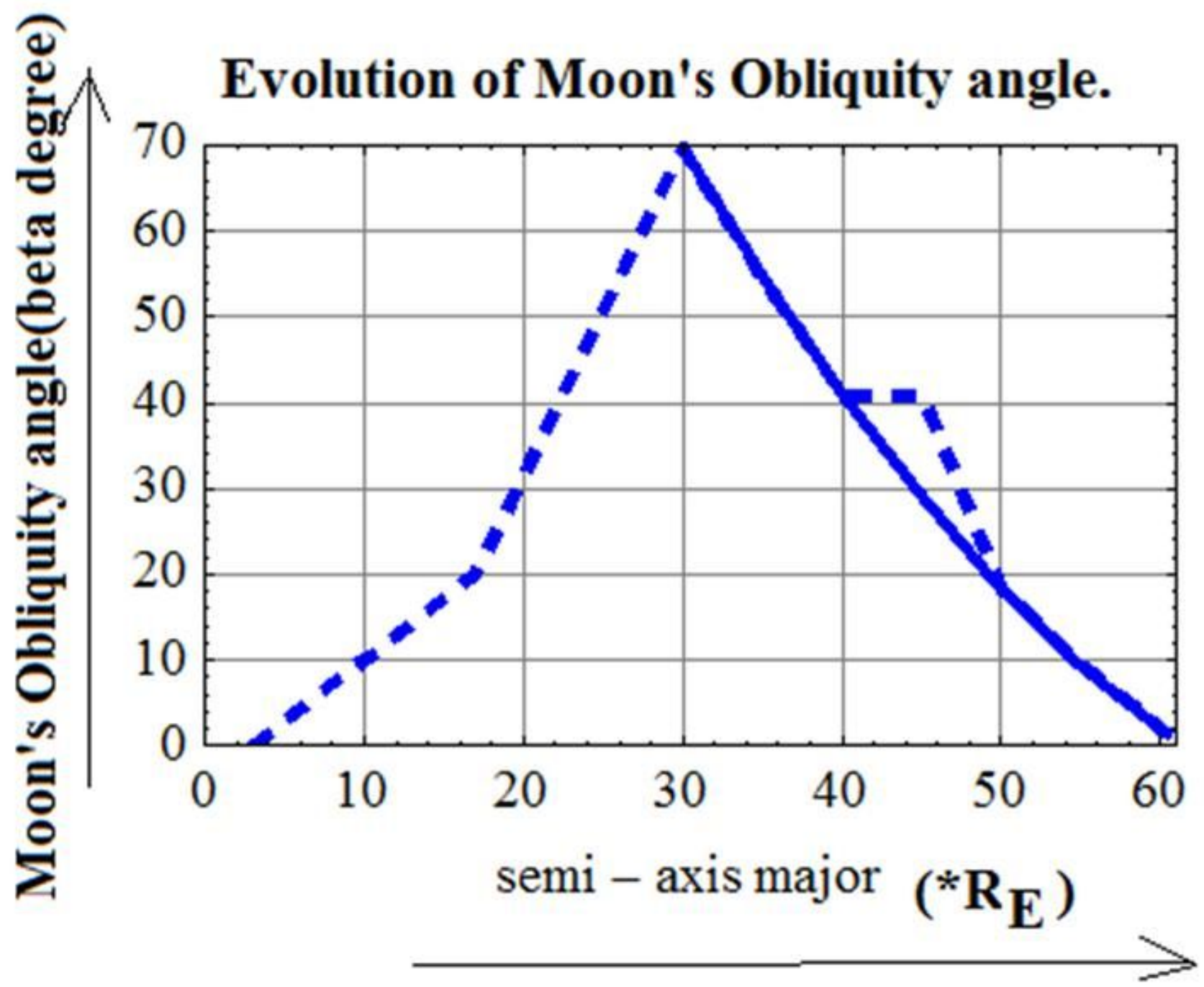

Figure 4

Moon's Obliquity $\left(\beta^{\circ}\right)$ based on AKM (bold blue) and based on Simulation results (dash blue) (Cuk et.al.2016)

\section{Supplementary Files}

This is a list of supplementary files associated with this preprint. Click to download.

- AKMSupportingInformationLettersv2.docx 